\title{
Morfea o esclerodermia localizada
}

\author{
Morphea or localized scleroderma
}

\section{Claudia Marcela Gaviria', Sol Beatriz Jiménez ${ }^{2}$, Jorge Gutiérrez ${ }^{3}$}

1. Médica, residente de Dermatología, Universidad CES, Medellín, Colombia

2. Médica dermatóloga; jefe, Servicio de Dermatología, Grupo Dermatología CES, Universidad CES, Medellín, Colombia

3. Médico dermatólogo; docente de Dermatología, Universidad CES, Medellín, Colombia

\section{Resumen}

La esclerodermia es un término que se deriva de las palabras griegas skleros (duro o endurecido) y dermis (piel).

La esclerodermia localizada, también conocida con el nombre de morfea, es un trastorno fibrosante raro que afecta la piel y los tejidos adyacentes, y se limita casi de manera exclusiva a los tejidos derivados del mesodermo. La patogénesis no está dilucidada por completo, pero se sabe que es una enfermedad autoinmunitaria en la cual existen múltiples factores que contribuyen a aumentar las citocinas proinflamatorias, lo cual conduce a un desequilibrio entre la producción y la destrucción del colágeno, que finalmente va a favorecer la fibrosis que caracteriza a esta enfermedad.

Aunque la morfea es un trastorno localizado de la piel, los pacientes con esta enfermedad comúnmente pueden tener síntomas sistémicos, como malestar general, fatiga, artralgias y mialgias, así como autoanticuerpos circulantes positivos.

Se diferencia principalmente de la esclerosis difusa (esclerosis sistémica) por la ausencia de esclerodactilia, fenómeno de Raynaud, cambios capilares del lecho ungular y compromiso de órganos internos.

PALABRAS ClAve: piel y enfermedades del tejido conjuntivo, esclerodermia, esclerodermia localizada.

\section{Summary}

Scleroderma is a term derived from the Greek words skleros (hard or indurated) and derma (skin).

Localized scleroderma, also known by the name of morphea, is a rare fibrosing disorder that affects the skin and adjacent tissues, and is limited almost exclusively to tissues derived from mesoderm. The pathogenesis is not fully elucidated, but it is known that results from an imbalance between the production and destruction of collagen.

Although morphea is a localized skin disorder, patients with this disease can often have systemic symptoms such as malaise, fatigue, arthralgias and myalgias, as well as positive self-circulating antibodies.

It differs mainly in systemic scleroderma (systemic sclerosis) by the absence of sclerodactyly, Raynaud's phenomenon, nailfold capillary changes and condition of internal organs.

KEY WORDS: Skin and connective tissue diseases, scleroderma, localized scleroderma.

\section{Correspondencia:}

Claudia Marcela Gaviria

Email:

claugavio2@hotmail.com

Recibido: 10 de marzo de 2014. Aceptado: 12 de mayo de 2014.

No se reportan conflictos de interés. 


\section{Introducción}

Al evaluar los trastornos esclerosantes de la piel nos encontramos con un sinnúmero de entidades, muchas de los cuales comprometen la piel y las estructuras adyacentes.

La esclerodermia se deriva de las palabras griegas skleros (duro o endurecido) y dermis (piel), y fue Hipócrates quien describió por primera vez esta condición ${ }^{1}$.

La esclerodermia se clasifica en: esclerodermia localizada o morfea, trastorno fibrosante que afecta la piel, el tejido celular subcutáneo, el hueso subyacente, rara vez, la cabeza y la cara, y puede afectar el sistema nervioso central; y esclerodermia sistémica que, al igual que la morfea, es una enfermedad fibrosante pero de compromiso sistémico, que se diferencia de la anterior por la ausencia de esclerodactilia, fenómeno de Raynaud, cambios capilares del lecho ungular y afección de los órganos internos ${ }^{2-4}$.

Este artículo trata de la esclerodermia localizada y, para evitar confusiones, nos referimos a esta como morfea.

\section{Epidemiología}

$\mathrm{Su}$ incidencia anual varía entre 0,4 y 2,7 por 100.000 personas, con un predominio femenino de 2,4 a 4,2:1 $\mathrm{y}$ es dos a tres veces más frecuente que la esclerosis sistémica ${ }^{4-6}$.

La prevalencia de esta enfermedad parece ser semejante tanto en niños como en adultos. En niños tiene un pico entre los 2 y los 14 años de edad, siendo la morfea lineal la forma de presentación más común, mientras que en los adultos la edad de presentación es entre los 40 y los 50 años, siendo la morfea en placas la forma clínica más común. La edad de presentación de las variantes menos frecuentes de la esclerosis localizada, es entre la tercera y la cuarta década de la vida ${ }^{2,4,7}$.

Aunque la morfea afecta a todas las razas, parece ser más prevalente en la raza blanca, que corresponde a 72,7 a $82 \%$ de los pacientes atendidos ${ }^{6,8}$.

Se considera que tanto los niños como los adultos con morfea son más propensos a tener historia familiar de morfea $\mathrm{u}$ otros trastornos autoinmunitarios, comparados con la población sana ${ }^{8}$.

\section{Fisiopatología}

Aunque la patogénesis de la morfea no está entendida por completo, es probable que se trate de un proceso multifactorial que conlleva un desequilibrio entre la producción y la destrucción de colágeno².

A pesar de las diferencias clínicas entre la esclerosis localizada y la sistémica, las características histopatológicas de las lesiones en piel son indistinguibles y ambas condiciones se caracterizan por presencia de un infiltrado de linfocitos $\mathrm{T}_{\mathrm{CD}}+$, exceso en la producción de colágeno de tipo I y III, y depósito de matriz extracelular. Además del aumento en la producción de colágeno, hay una cierta inhibición en la degradación de esta molécula debido a la presencia de autoanticuerpos dirigidos contra la colagenasa MMP-1. Finalmente, el daño de los vasos sanguíneos pequeños, la activación de los linfocitos $\mathrm{T}$ y la alteración de la producción de tejido conjuntivo, constituyen los tres componentes principales de la esclerosis $^{2,9,10}$.

El proceso por el cual ocurre esto aún permanece desconocido, pero se cree que todo se inicia por una lesión vascular por una infección, asociada a la exposición ambiental y, posiblemente, por la presencia de autoanticuerpos contra las células endoteliales determinados genéticamente.

Esta lesión endotelial libera citocinas que aumentan la expresión de la molécula de adhesión celular vascular 1 (VCAM-1), la molécula de adhesión intercelular 1 (ICAM-1), y la E-selectina, las cuales van a reclutar células $\mathrm{T}$ que producen citocinas profibróticas, como la interleucina 4, la interleucina 6 y el factor de crecimiento transformante beta (TGFb); estas citocinas también reclutan eosinófilos, células T CD41 y macrófagos; se cree que estas células presentadoras de antígenos presentan autoantígenos que llevan a la formación de autoanticuerpos ${ }^{2}$.

La regulación positiva de varias moléculas de adhesión, como el TGFb, el factor de crecimiento derivado de plaquetas, el factor de crecimiento tisular y las interleucinas 4, 6 y 8, aumenta la producción de colágeno, fibronectina y proteoglucanos y, por otro lado, disminuye la producción de proteasas y aumenta su inhibición. Este desequilibrio entre producción y degradación de colágeno, que se refleja en el aumento de la producción de colágeno y la disminución de las metaloproteinasas de matriz (MMP) responsables de la degradación del colágeno, es la piedra angular de la fibrosis que caracteriza a la morfea. Se ha postulado que los pacientes con morfea o con esclerosis sistémica tienen autoanticuerpos que son capaces de inhibir la actividad de colagenasa de la MMP1; estos anticuerpos anti-MMP1 no se ven en los controles sanos ni en los pacientes con lupus eritematoso sistémico o dermatomiositis y, por lo tanto, pueden ser específicos de la esclerosis. Por esta razón, la inhibición de la degradación del colágeno en estos casos puede también ser parcialmente mediada por anticuerpos anti-MMP $2,10,11$.

Además de lo anterior, la literatura científica sugiere que las citocinas de perfil Th1, Th2 y Th17 contribuyen 
a la patogénesis. Hay un concepto general de que las citocinas Th1 proinflamatoria y Th17 están elevadas durante las primeras etapas de la esclerodermia, mientras que las citocinas Th2 se correlacionan principalmente con el grado de fibrosis y las complicaciones de la enfermedad; aunque esto es cierto en la esclerosis sistémica, sólo ha sido parcialmente investigado en la esclerodermia localizada ${ }^{12}$.

\section{Factores de riesgo}

Los siguientes factores se han asociado con el desarrollo de la enfermedad: trauma, radiación, medicamentos, infecciones, historia familiar y autoinmunidad ${ }^{2,7}$.

\section{Trauma}

En dos revisiones retrospectivas de 886 pacientes con morfea, se reportó un trauma mecánico previo en, aproximadamente, el $13 \%$ de los niños ${ }^{6,13}$.

Hay, además, reportados en la literatura científica, ocho casos de morfea después de vacunas de sarampión, paperas y rubéola, difteria, tétano y tos ferina, BCG (bacilo Calmette-Guerin) y hepatitis B. Por la gran variedad de las vacunas implicadas, se cree que es el trauma de la vacunación o probablemente sus conservantes los que promueven el desarrollo de morfea, en lugar de la vacuna en sí. El trauma de la inyección puede inducir un proceso de cicatrización excesivo que produce como consecuencia una fibrosis acentuada ${ }^{2}$.

\section{Radiación}

La incidencia de morfea después de radiación ha sido estimada en uno de cada 500 pacientes ${ }^{14}$. Las mujeres que reciben radioterapia para el tratamiento de cáncer de mama son las que tienen el mayor riesgo; en esta población, este efecto se presenta tardíamente, generalmente, un año después del final del tratamiento ${ }^{14,15}$.

La morfea inducida por la radiación se desarrolla comúnmente en la misma área de la irradiación; sin embargo, entre el 20 y el $25 \%$ de los casos se puede extender más allá del campo irradiado o presentarse en un lugar distante ${ }^{14}$.

\section{Medicamentos}

Se ha observado que múltiples medicamentos se asocian con el desarrollo de lesiones similares a la morfea, entre los cuales están los siguientes: bisoprolol, bleomicina, D-penicilamina, vitamina K1, bromocriptina, peplomicina, L-5-hidroxitriptófano en combinación con carbidopa, pentazocina y balicatib ${ }^{7,16}$. Las lesiones cutáneas aparecieron después de un período medio de
12,5 meses (rango entre 1 y 36 meses). Estos medicamentos pueden predisponer a la formación de autoanticuerpos que lesionan los capilares, o los pueden lesionar directamente, lo que resulta en la formación de lesiones de morfea ${ }^{16}$.

\section{Infección}

En 1986, Asbrink, et al., observaron que el $12 \%$ de sus pacientes con acrodermatitis crónica atrófica también presentaban lesiones de liquen escleroso y atrófico ${ }^{2}$. Estos hallazgos, asociados a las similitudes clínicas e histológicas entre el liquen esclero-atrófico y la morfea, llevó a buscar la presencia de Borrelia burgdorferi en esta última entidad, como lo demuestra el estudio de Eisendle, et al., quienes concluyeron que existe una participación específica de esta bacteria o de otras especies, desencadenantes del desarrollo de esta enfermedad ${ }^{17}$.

Sin embargo, a pesar de los resultados que han arrojado estos estudios, el papel de este microorganismo en la morfea es confuso y, hasta el momento, hay poca evidencia y no es categórica la relación entre Borrelia y morfea ${ }^{2,10}$.

\section{Autoinmunidad}

De todos los niños con morfea, entre el 2 y el $5 \%$ tiene una enfermedad autoinmunitaria asociada, sea vitiligo, tiroiditis de Hashimoto, enfermedad de Graves, diabetes mellitus de tipo 1 o colitis ulcerativa, lo cual sugiere un componente genético; sin embargo, los genes de vulnerabilidad a estas entidades y para la esclerosis localizada todavía se desconocen $(7,8,13)$. Por otro lado, se estima que, aproximadamente, el $30 \%$ de los adultos con morfea tienen una enfermedad autoinmunitaria asociada; estos porcentajes son bastante elevados con respecto a la población general, lo que apoya aún más el papel de la autoinmunidad en el desarrollo de la patogénesis de la morfea ${ }^{8}$.

En una revisión retrospectiva de 245 pacientes con morfea, se evaluó la frecuencia de las manifestaciones extracutáneas y de autoinmunidad, y se demostró que tanto los niños como los adultos estaban afectados casi por igual por estas condiciones. La prevalencia de la autoinmunidad asociada a la morfea generalizada fue mucho mayor que la encontrada en las demás variantes. La frecuencia de antecedentes familiares de enfermedad autoinmunitaria mostró una tendencia a favor de las variantes generalizada y mixta. Mientras que las manifestaciones sistémicas fueron más comunes en la morfea generalizada, el subtipo lineal mostró una asociación significativa con manifestaciones neurológicas. Los anticuerpos antinucleares fueron positivos con mayor frecuencia en la morfea generalizada y mixta ${ }^{8}$. 


\section{"La clasificación de esta enfermedad}

\section{ha sido muy discutida y aún los autores} difieren para establecer de manera definitiva alguna clasificación".

\section{Clasificación}

La clasificación de esta entidad se basa en su forma de presentación clínica. Peterson, et al., en 1995, fueron los que informaron inicialmente sobre esta clasificación, describiendo cinco tipos clínicos: morfea en placa, que incluye morfea en placa, guttata, atrofodermia de Pasini y Pierini, queloides, liquen escleroso y atrófico; morfea generalizada, que afecta más de dos zonas del cuerpo; morfea ampollosa; morfea lineal, que incluye compromiso de las extremidades, morfea en golpe de sable y atrofia hemifacial progresiva; y morfea profunda, que incluye morfea profunda o subcutánea, fascitis eosinofílica y morfea panesclerótica ${ }^{18}$.

Sin embargo, este sistema de clasificación ha sido muy discutido porque incluye muchos diagnósticos que no están en el mismo espectro de la morfea, como la atrofodermia de Pasini y Pierini, el liquen escleroso y atrófico y la fascitis eosinofílica y, además, esta clasificación no incluye la forma de presentación mixta que corresponde a la manifestación clínica de dos o más tipos de variantes simultáneamente ${ }^{2,4}$.

En 2004, un grupo de la Paediatric Rheumatology European Society hizo una nueva propuesta de clasificación de la esclerodermia juvenil localizada, para corregir algunas carencias de la clasificación anterior propuesta por Peterson. Excluyen de esta clasificación condiciones como la atrofodermia de Pasini y Pierini, el liquen escleroso y atrófico, y la fascitis eosinofílica, e incluyen el concepto de morfea mixta para identificar aquellos pacientes que presentan una combinación de dos o más tipos de lesiones, ya que hasta el $15 \%$ de los niños pueden presentarla ${ }^{4,19}$. La clasificación que propone este grupo y que es extrapolable a los adultos, es la siguiente: morfea circunscrita o en placas, que incluye la variante superficial y profunda; morfea lineal, la cual se puede presentar en el tronco, extremidades o cabeza; morfea generalizada; morfea panesclerótica, y morfea mixta ${ }^{4,19}$.

Recientemente, en 2009, un grupo de expertos alemanes establecieron una nueva clasificación teniendo en cuenta la extensión y profundidad de la fibrosis; diferencia, además, las formas limitadas, generalizadas, lineal y profunda junto con sus subtipos asociados. El tipo limitado incluye la morfea en placas, la morfea guttata y la atrofodermia idiopática de Pasini y Pierini; el tipo generalizado incluye la esclerodermia localizada generalizada, la morfea panesclerótica y la fascitis eosinofílica (síndrome de Shulman); el tipo Lineal incluye la esclerodermia lineal localizada de las extremidades, la esclerodermia lineal localizada en golpe de sable y la hemiatrofia facial progresiva o síndrome de Parry Romberg, y el tipo profundo que incluye la morfea profunda ${ }^{20}$.

La clasificación de esta enfermedad ha sido muy discutida y aún los autores difieren para establecer de manera definitiva alguna clasificación. En este artículo se hace referencia a la planteada en el 2004, la cual según los autores es extrapolable a la población adulta y es un esquema sencillo que no presenta los puntos debatidos en las demás clasificaciones.

\section{Presentación clínica}

La lesiones de morfea inicialmente tienen una fase inflamatoria o activa que corresponde clínicamente a placas eritematosas o violáceas oscuras. Con el tiempo, el centro de estas lesiones se torna blanco y esclerótico, y característicamente los bordes adquieren una conformación en anillo de color violáceo. Después de la fase 
activa de la enfermedad, el daño resultante se manifiesta como placas hipopigmentadas o hiperpigmentadas posinflamatorias escleróticas, generalmente sin anexos pilosos y anhidróticas, esto secundario al abundante depósito de colágeno, el cual destruye los folículos pilosos y demás anexos epidérmicos perilesionales.

Estas lesiones comprometen principalmente tronco, mamas, extremidades y el área entre la cadera y la región inguinal. Las manos, los pies y la cabeza, por lo general, están respetados ${ }^{2,11}$.

\section{MORFEA CIRCUNSCRITA O MORFEA EN PLACA: es} la variante más común, se presenta con menos de tres placas que se localizan principalmente en el tronco. Este tipo de morfea, a su vez, puede ser superficial o profunda. La variante superficial es más común, su compromiso se limita a la epidermis y a la dermis, y las placas iniciales tienen un borde inflamatorio o violáceo, aunque algunas lesiones se pueden presentar como máculas eritematosas o hiperpigmentadas de color café sin induración. La variante profunda (anteriormente denominada morfea subcutánea), por el contrario, afecta la dermis y el tejido subcutáneo, y puede o no comprometer la fascia subyacente y el músculo. La piel supraadyacente puede no estar comprometida, o presentarse como una piel atrófica y endurecida. Los pacientes adultos con este tipo de morfea pueden desarrollar placas en zonas de presión, como la cadera, la cintura y la región inframamaria (respetando uniformemente los pezones), lo que corresponde a un fenómeno de Koebner ${ }^{2,4,11}$.

A pesar de que en muchos casos puede haber períodos prolongados de inactividad de la enfermedad, los pacientes con morfea son propensos a desarrollar nuevas lesiones durante toda su vida ${ }^{2,4,11}$.

2. MORFEA GENERALIZADA: es una variante rara, ocurre en 7 a $9 \%$ de los pacientes con morfea. Se define como más de cuatro placas induradas mayores de $3 \mathrm{~cm}$ o que afecten dos o más partes del cuerpo, respetando cara y manos. Clínicamente, se presenta como placas ligeramente inflamadas, pigmentadas, mal definidas, la piel se palpa engrosada y adherida a los planos profundos, generalmente se localizan en el tronco, pero puede afectar otras áreas como las extremidades. Los signos de inflamación aguda, como edema y eritema, suelen estar ausentes ${ }^{4,11}$. El compromiso de la piel en esta variante se limita generalmente a la dermis, y rara vez afecta el tejido subcutáneo ${ }^{2}$.

Estos pacientes tienen mayor tendencia a presentar síntomas sistémicos, como fatiga, mialgias y artralgias, y mayor predisposición a tener serología positiva para autoanticuerpos, especialmente para los anticuerpos antinucleares (ANA) ${ }^{2,8,13}$. Además, es usual detectar eosi- nofilia periférica, anti-ADN de cadena simple, hipocomplementemia e incluso anticuerpos antifosfolípidos ${ }^{4}$. Por estas y otras razones, es difícil diferenciar la morfea generalizada de la esclerosis sistémica. Sin embargo, los pacientes con morfea generalizada no presentan fenómeno de Raynaud, esclerodactilia o cambios capilares en el lecho ungular; la cara suele respetarse y, aunque los cambios esclerosos de la piel pueden ser muy extensos en la morfea generalizada, no suele presentarse la facies típica de la esclerodermia sistémica, como la desaparición de las arrugas de expresión y el adelgazamiento de los labios ${ }^{2,4}$.

3. MORFEA LINEAL: es la variante más común en niños y afecta entre el 41,8 y el $67 \%$ de ellos $(4,6,8)$. Generalmente, se presenta como una lesión única, unilateral y de distribución lineal, que se localiza en cara, cuero cabelludo (área fronto-parietal) o extremidades. Muchas veces estas lesiones lineales siguen las líneas de Blaschko, por lo que se ha sugerido un mosaico como factor contribuyente en la patogénesis de esta variante $(2,11)$. Así, como se demuestra en un estudio retrospectivo que incluyó 65 niños con morfea lineal, cuyo objetivo fue identificar patrones comunes de presentación clínica en niños con morfea lineal y establecer si la morfea lineal seguía o no las líneas de Blaschko, se encontró que la morfea lineal sigue francamente las líneas de Blaschko y se postula un mosaico en los pacientes propensas a desarrollar este tipo de morfea y la exposición a algún factor desencadenante puede resultar en su desarrollo ${ }^{21}$.

Los estudios han demostrado que entre el 5 y el $25 \%$ de los niños con enfermedad lineal pueden tener compromiso bilateral ${ }^{2,6}$.

Esta variante, a su vez, tiene varias formas de presentación, y las tres más comúnmente descritas son: morfea lineal que compromete las extremidades, morfea en golpe de sable y hemiatrofia facial progresiva.

Morfea lineal que compromete extremidades: son placas atróficas lineales profundas, mal definidas, con alteración de la pigmentación, que interfieren con el crecimiento de la extremidad produciendo divergencia de longitud entre ambas extremidades, deformidades y contracturas articulares, esto producido por la atrofia del músculo y el hueso subyacente ${ }^{2,4}$.

Morfea en golpe de sable: se presenta generalmente en la línea media de la frente, pero también puede comprometer el cuero cabelludo en forma de placas alopécicas atróficas en patrón lineal, y la cara siendo las mejillas, la nariz, el labio superior y los ojos las áreas más afectadas. El compromiso más temido es el del sistema nervioso central.

El carácter unilateral de estas lesiones, su preferencia 
por la región parietal y la tendencia a deformar el hueso dando lugar a lesiones deprimidas, han llevado a términos tan descriptivos como el de esclerodermia en coup de sabre ${ }^{2,4}$.

Hemiatrofia facial progresiva: conocida también como síndrome de Parry-Romberg, se caracteriza por atrofia facial unilateral que afecta la piel, el tejido subcutáneo, la grasa, el músculo y en algunas ocasiones estructuras osteocartilaginosas, lo que en la clínica se traduce en cambios mínimos o ausentes en la superficie cutánea, pero sí en atrofia y deformidades faciales. Generalmente es de resolución espontánea y compromete preferentemente los dermatomas de una o varias ramas del quinto par craneal. Esto favorece la aparición de alteraciones oculares como endoftalmos, uveítis, parálisis de la musculatura ocular, ptosis o síndrome de Horner; y deformaciones de la mandíbula con la subsecuente mala oclusión dental, implantación inadecuada de los dientes, atrofia de las raíces o retraso en la aparición de los dientes. Este trastorno predomina en mujeres y se presenta generalmente en la primera o segunda década de la vida, progresa lentamente en 2 a 20 años para luego entrar en una fase estacionaria o de meseta ${ }^{2,4,22,23}$.

4. MORFEA PANESCLERÓTICA: variante inusual, es la forma más debilitante de la enfermedad. Generalmente se presenta en la infancia, aunque también se ha descrito en adultos. Es típico que las placas se localicen en las superficies extensoras de las extremidades y el tronco, respeta la punta de los dedos de las manos y los pies, y no hay presencia de fenómeno de Raynaud. La clínica es similar a la de la morfea generalizada, pero en este caso se encuentra un compromiso mayor y mucho más extenso no solo de la piel y el tejido celular subcutáneo, sino también, de estructuras más profundas como los músculos, los tendones y los huesos, lo cual lleva a una gran morbilidad producida por la atrofia muscular, las contracturas articulares y las úlceras que no cicatrizan ${ }^{2,4}$. Además, hay mayor riesgo de desarrollar carcinoma escamocelular sobre las placas panescleróticas de larga evolución ${ }^{2,24,25}$. Wollina, et al., estiman que anualmente $6,7 \%$ de los pacientes con este tipo de morfea desarrollan carcinoma escamocelular ${ }^{26}$.

5. MORFEA MIXTA: se produce hasta en el $15 \%$ de los pacientes con morfea y es una combinación de dos o más de las variantes anteriormente descritas ${ }^{2,13}$.

\section{Manifestaciones sistémicas}

La morfea no tiene repercusión sistémica grave, salvo algunas excepciones, pero sí puede ser causa de una gran morbilidad ya que las lesiones, sobre todo las localizadas en cara y extremidades, pueden ser deformantes y generar una gran limitación funcional. Además, entre más extensa y profunda sea la afección del proceso fibroso, aumenta la probabilidad de detectar alguna alteración en órganos internos. Por esta razón, las manifestaciones extracutáneas de la morfea son más comunes en la morfea en placas variante profunda, la lineal y la generalizada, y entre las m anifestaciones están la fatiga, las mialgias y las artralgias ${ }^{4,11}$.

Se ha documentado la frecuencia de las complicaciones en la esclerodermia lineal, sobre todo cuando es en golpe de sable o en la hemiatrofia facial progresiva, así:

Neurológicas: se encuentran casi en el $20 \%$ de estos pacientes $^{13,23,27}$, y entre estas se destacan la epilepsia, la migraña, las neuralgias, las parestesias de diversos pares craneales y las alteraciones electroencefalográficas y en diversas pruebas de imagen. Así como lo demuestra en un estudio publicado en el 2003, en el cual se evaluó la presencia de compromiso del sistema nervioso central en la hemiatrofia facial progresiva y la esclerodermia en golpe de sable en 26 pacientes, se concluyó que la afección del sistema nervioso central es frecuente en los pacientes con hemiatrofia facial progresiva, independientemente de la fecha de presentación, de la induración cutánea y de si hay placas coexistentes de esclerodermia localizada en otras áreas ${ }^{27}$.

Oftalmológicas: ocurren hasta en el $15 \%$, predominando la esclerosis de las estructuras de los anexos, seguida de la inflamación del segmento anterior y la uveítis anterior. Se ha demostrado en estos pacientes, un mayor riesgo de asociación con otras complicaciones extracutáneas, sobre todo las neurológicas, como se describe en un estudio publicado en el 2007, en el cual recolectaron datos de 750 niños con esclerodermia localizada, para analizar el compromiso ocular de estos pacientes. Encontraron que, de 750 pacientes, 24 (3,2 \%), 16 con esclerodermia en golpe de sable, 5 con morfea lineal, 2 con morfea generalizada y 1 con morfea en placas, tenían una afectación ocular significativa. Concluyeron que las alteraciones oculares no son infrecuentes en la esclerodermia localizada juvenil, especialmente en la variante en golpe de sable y, además, estas alteraciones se asocian frecuentemente con afectación de otros órganos internos, particularmente del sistema nervioso central. Sugieren hacer seguimiento oftalmológico a todos los pacientes con esclerodermia localizada juvenil, pero lo consideran obligatorio en los con que presentan lesiones concomitantes en cara o afectación del sistema nervioso central $^{28}$. Por eso, se recomienda seguimiento oftalmológico cada tres a cuatro meses durante los primeros tres años, cuando existe compromiso facial lineal ${ }^{2}$. 
Alteraciones autoinmunitarias: los adultos (10,6 \%) y los niños (12 a 23,8 \%) con morfea tienen una mayor prevalencia de trastornos autoinmunitarios en sus familias, en comparación con la población general ${ }^{2,8,13}$. El $1 \%$ de los pacientes tiene un familiar en primero o segundo grado de consanguinidad con esta enfermedad ${ }^{8}$.

Psicológicos: tanto los niños como los adultos con morfea tienen niveles más altos de depresión y ansiedad que los individuos sanos de su misma edad ${ }^{2}$.

\section{Diagnóstico}

La evaluación histológica es lo más importante en el diagnóstico de la esclerodermia localizada, pero es claro que la diferenciación histopatológica entre morfea y esclerosis sistémica no es fácil, aunque existen algunos hallazgos que pudieran ayudar; esta diferenciación no es categórica y, por el contrario, los hallazgos generales son muy similares.

En un estudio publicado en 1998 con el fin de diferenciar histopatológicamente estas dos entidades, se evaluaron 51 pacientes (32 con esclerodermia localizada y 19 con esclerosis sistémica) y se observó que la localizada y la sistémica se pueden diferenciar, por lo general, por la distribución y la densidad del infiltrado inflamatorio y por una dermis papilar comprometida o indemne. Se observó que los cambios inflamatorios y la esclerosis de la dermis papilar eran más prominentes en la esclerodermia localizada, y estaba ausente en la sistémica. También, reportaron diferencias significativas en cuanto al espesor de la dermis: estaba notablemente engrosada en el $44 \%$ de los pacientes con esclerodermia localizada y moderadamente engrosada en el $48 \%$ de aquellos con esclerosis sistémica. Además, se observó que los haces gruesos de colágeno se encuentran principalmente en la parte baja de la dermis reticular y en el panículo adiposo en el caso de la esclerosis sistémica, mientras que en la esclerodermia localizada se encuentran en todas las capas de la dermis. Se evidenció que la duración de la enfermedad no se correlacionaba con los cambios histopatológicos en la esclerosis sistémica, mientras que, en todos los casos de la forma localizada con esclerosis en la dermis papilar, la duración de la enfermedad fue superior a un año; sin embargo, la densidad y la distribución del proceso inflamatorio no mostraron correlación con la duración de la lesión individual en la esclerodermia localizada ${ }^{29}$.

Según las etapas de la enfermedad, se han descrito los siguientes cambios para ambas entidades ya que, como se mencionó anteriormente, son difíciles de diferenciar histológicamente: en la etapa inicial tanto de la morfea como de la esclerosis sistémica, en la dermis reticular se observa un infiltrado perivascular linfocitario con escasas células plasmáticas y eosinófilos, las células endoteliales se ven con edema y los haces de colágeno pueden presentar engrosamiento; mientras que en la etapa final, el infiltrado inflamatorio desaparece y los haces de colágeno de la dermis reticular son prominentemente eosinofílicos, engrosados y hacinados, extendiéndose hasta el tejido subcutáneo, las glándulas ecrinas se tornan atróficas a causa del colágeno grueso que las rodea, hay una disminución significativa de los vasos sanguíneos, y la grasa subcutánea parece "atrapada” en la dermis debido a la extensión de colágeno en los tejidos subcutáneos ${ }^{2,7,11}$.

Como se describió anteriormente, la morfea se presenta con haces de colágeno completamente engrosados localizados en la dermis y pérdida de dendrocitos dérmicos $\mathrm{CD}_{34}{ }^{+}$, pero solo hasta el 2009 se llevó a cabo un estudio sobre el patrón de las fibras elásticas, correlacionándolo con la expresión de $\mathrm{CD}_{34}$ en esta entidad. En este estudio se confirmó la preservación y disposición recta, paralela y compacta de las fibras elásticas y, además, se sugirió que la inmunohistoquímica de $\mathrm{CD}_{34}$ puede servir como herramienta para el diagnóstico de esta enfermedad ${ }^{30}$.

En cuanto a la profundidad del compromiso de los diferentes tipos de morfea, se puede decir que el proceso fibroso de la morfea en placas, variante superficial, se confina a la dermis, mientras que, en la morfea en placas, variante profunda, la fibrosis afecta la dermis profunda, el tejido adiposo y el músculo; no obstante, a diferencia de la morfea lineal, las lesiones son más difusas, no siguen un patrón lineal y, generalmente, no comprometen el hueso como sí puede llegar a suceder en la lineal ${ }^{4}$.

\section{Exámenes}

\section{Laboratorio}

Por lo general, los hallazgos de laboratorio son muy inespecíficos y no se requieren de rutina; a veces, en el hemograma se puede encontrar eosinofilia, y se puede encontrar ANA y anti-ADN positivos, aunque estos últimos con menor frecuencia ${ }^{10}$.

En cuando a las características serológicas, en la esclerosis sistémica existen anticuerpos muy específicos: en la esclerosis sistémica limitada se encuentran los anticuerpos anticentrómero y en la difusa se encuentran los anticuerpos anti-Scl 70. Estos hallazgos no se observan en la esclerodermia localizada; sin embargo, sí hay hallazgos de autoinmunidad en los exámenes de 
laboratorio ${ }^{7}$. Esto se demostró en el estudio de Takehara y Sato, en el cual los autoanticuerpos más frecuentes (73\%) en la morfea fueron los ANA en patrón homogéneo (detectados por IFI) en y los anticuerpos antitopoisomerasa II alfa $(76 \%)^{3}$. Sin embargo, la prevalencia de los títulos positivos de ANA en la morfea oscilan entre el 20 y el $80 \%$, pero los títulos no parecen correlacionarse con la evolución de la enfermedad ${ }^{3,6,8}$.

Los anticuerpos antitopoisomerasa II alfa son mucho más frecuentes en la morfea que en otras enfermedades autoinmunitarias, como la esclerosis sistémica (14\%), el lupus eritematoso sistémico (8\%) y la diabetes mellitus $(10 \%)^{2}$.

En la morfea lineal de las extremidades, asociada con compromiso articular, se puede encontrar aumento de los niveles del factor reumatoideo, pero este valor puede no correlacionarse con la gravedad de la artritis ${ }^{31,32}$.

\section{Estudios de imágenes}

La morfea circunscrita o en placas no requiere un examen radiológico de rutina, mientras que en la morfea en golpe de sable o la hemiatrofia facial progresiva que se presenta más frecuentemente en niños, se debe considerar la resonancia magnética cerebral para detectar el posible compromiso del sistema nervioso central, y las calcificaciones subcorticales y la atrofia cerebral los hallazgos más comunes ${ }^{6,33}$.

\section{Diagnóstico diferencial}

La esclerodermia localizada tiene un amplio espectro de subtipos y etapas clínicas, razón por la cual se debe considerar una variedad de diagnósticos diferenciales a la hora de abordar un paciente con sospecha clínica de esta entidad.

La principal diferenciación se debe hacer con la esclerosis sistémica. Después de hacerlo, los diagnósticos diferenciales que se deben tener en cuenta para la morfea circunscrita o en placas son el liquen escleroso extragenital y la acrodermatitis crónica atrófica; para la morfea generalizada, la enfermedad crónica de injerto contra huésped, la esclerosis sistémica y la fibrosis sistémica nefrogénica; y para la morfea lineal, la paniculitis lúpica y otras paniculitis ${ }^{\text {. }}$.

Otros diagnósticos diferenciales para tener en cuenta en un paciente con antecedentes de radiación y lesiones clínicamente similares a una morfea, son el carcinoma escamocelular y la radiodermitis, casos en los cuales en la mayoría de las veces es necesario obtener una biopsia de piel para llegar al diagnóstico definitivo ${ }^{14,15}$.

\section{Medición de la efectividad de los tratamientos}

\section{Parámetros clínicos}

Se han utilizados múltiples sistemas de puntuación clínica para evaluar las tasas de éxito después del tratamiento. Sin embargo, por la difícil estandarización de estos métodos y la dificultad para definir la mejoría clínica, estos métodos son poco usados.

Varios grupos de investigación, en los estudios piloto de tratamiento de la esclerodermia localizada, han utilizado una puntuación de la piel no validada (Modified Skin Score, MSS). En esta puntuación se divide el cuerpo en siete regiones anatómicas y cada región se califica de o a 3 para dos ítems: grado de engrosamiento y porcentaje de compromiso. Otro sistema de puntuación, el índice de gravedad de la esclerodermia localizada en la piel (Localized Scleroderma Skin Severity Index, LoSSI), ha sido validado para esta entidad. En este método se divide el cuerpo en 18 áreas anatómicas y se mide el eritema de la piel, el grosor y las nuevas lesiones, en una escala de o a $3^{34}$.

Recientemente, se validó el índice de daño en la piel por esclerodermia localizada (Localized Scleroderma Skin Damage Index, LoSDI), el cual ha demostrado gran fiabilidad. Arkachaisri, et al., recomiendan combinar la escala LoSSI, la LoSDI y la evaluación global del médico, para medir la actividad y el daño en la esclerodermia localizada. Esto compone la herramienta de evaluación para la esclerodermia localizada cutánea (Localized Scleroderma Cutaneous Assessment Tool, LoSCAT), una puntuación combinada que sigue el modelo de una herramienta bien establecida para el lupus eritematoso cutáneo (Cutaneous Lupus Erythematosus Disease Area and Severity Index, CLASI). Al igual que el CLASI, el LoSCAT podría convertirse en una herramienta estándar para evaluar la afección de la piel en la morfea ${ }^{35}$.

Parámetros imaginológicos: se ha estudiado una variedad de métodos imaginológicos como medida adyuvante para evaluar la respuesta y el seguimiento. La actividad de la lesión con frecuencia se evalúa por termografía y láser Doppler, teniendo presente que en las zonas activas y de nuevas lesiones, la temperatura y el flujo de la sangre se incrementan. En el 2007 se publicó un estudio en el que se concluyó que la flujometría láser Doppler es una técnica útil, no invasiva, que sirve para evaluar la actividad de la morfea en niños y es más precisa que la termografía para este fin ${ }^{36}$. Zulian, et al., publicaron en el mismo año un nuevo método llamado "puntuación computadorizada de piel" (Com- 


\section{"El tratamiento está dirigido, tanto a la} fase activa, con el objetivo de estabilizar el tamaño de las lesiones actuales, como a la fase inactiva, para prevenir futuras cicatrices y contracturas".

puterized Skin Score, CSS), el cual mide el endurecimiento y la inflamación del área afectada mediante un software especializado y, además, es capaz de calcular la superficie de piel afectada, el borde inflamatorio y el centro esclerótico ${ }^{37}$.

\section{Tratamiento}

No existe un tratamiento realmente eficaz y universal, por lo que es importante evaluar en forma correcta la extensión y la gravedad de la enfermedad, antes de tomar una decisión terapéutica.

El tratamiento está dirigido, tanto a la fase activa, con el objetivo de estabilizar el tamaño de las lesiones actuales, como a la fase inactiva, para prevenir futuras cicatrices y contracturas ${ }^{38}$.

Sin embargo, por tratarse de una entidad relativamente rara, es difícil llevar a cabo estudios controlados de asignación aleatoria, por lo cual son pocas las opciones de tratamiento basadas en la evidencia. Además, la mejoría clínica es difícil de establecer por la falta de una herramienta validada y estandarizada para este fin.

Para fines prácticos, es adecuado dividir las opciones terapéuticas en tópica, sistémica, física (fototerapia) y las nuevas alternativas, como los productos biológicos y los rellenos ${ }^{34,38}$.

\section{Tópico}

Tacrolimus: es uno de los agentes más utilizados en esta entidad. Se cree que el mecanismo por el cual induce regresión de las lesiones esclerodermiformes, se debe a su papel inmunomodulador y antiinflamatorio, el cual está vinculado a la inhibición de la activación de los linfocitos $\mathrm{T}$ y a la disminución en la producción de citocinas inflamatorias ${ }^{39}$.

En un estudio publicado en el 2005, que incluyó siete pacientes con diagnóstico de morfea, se evaluó la eficacia del tacrolimus tópico al o,1\%, dos veces al día, durante tres meses y con seguimiento cada cuatro semanas. Al finalizar el tratamiento, las lesiones tempranas mostraron una piel normal o casi normal. Estos resultados sugieren que el tacrolimus tópico puede ser una opción de tratamiento, especialmente por su gran tolerabilidad, que permite su uso prolongado sin efectos secundarios locales o sistémicos ${ }^{39}$.

En el 2008, se publicó un estudio que incluyó trece pacientes con placas de morfea. Se evaluó la eficacia del tacrolimus en ungüento al o,1 \% durante cuatro meses y con seguimiento máximo de un año. Cuatro pacientes presentaron una mejoría menor del $25 \%$, dos mostraron mejoría entre 50 y $70 \%$, y los siete restantes mostraron mejoría de $70 \%$. En los pacientes con placas gruesas, la respuesta clínica fue mala, en comparación con los que presentaban placas menos gruesas y más eritematosas. En este estudio se concluyó que el tacrolimus tópico al o,1 \% se puede usar en la morfea, especialmente en las lesiones inflamatorias tempranas, incluso como tratamiento de primera línea, con buena efectividad y tolerabilidad ${ }^{40}$.

Kroft, et al., publicaron en el 2009 un estudio de asignación aleatoria y controlado con placebo, cuyo objetivo fue evaluar la efectividad del tacrolimus en ungüento al $0,1 \%$ aplicado dos veces al día durante doce semanas, en diez casos de morfea activa. Se observó que los pa- 
cientes tratados con tacrolimus presentaban mejoría clínica y disminución significativa de la induración de las lesiones. Los autores concluyeron que era el primer estudio piloto doble ciego, controlado con placebo, en el que se había demostrado que el tacrolimus era un tratamiento eficaz para la fase activa de esta condición ${ }^{41}$.

Calcipotriol: sus efectos como inmunomodulador y como regulador en la proliferación de fibroblastos y en la síntesis de colágeno, se han estudiado numerosas veces in vitro e in vivo, sustentando su uso en esta entidad. Además, se ha visto que inhibe indirectamente la secreción de interleucina 2 (cuya concentración sérica se aumenta en la morfea) al inhibir las células T activadas. Esto apoya que el calcipotrieno puede suprimir la función de estas células ${ }^{42}$.

En un estudio abierto que incluyó doce pacientes con morfea, se evaluaron la eficacia y seguridad del calcipotriol en ungüento al o,005 \%, dos veces al día durante tres meses. Todos los pacientes presentaron una mejoría clínica estadísticamente significativa, sin detectarse efectos adversos ni alteraciones de laboratorio, concluyéndose que es un tratamiento eficaz para esta entidad, pero se requieren de estudios controlados para confirmar estos resultados ${ }^{42}$.

En un reporte de caso publicado en el 2003, se demostró la eficacia del calcipotriol, aplicado dos veces al día durante tres meses, en una niña de cinco años después de una falla terapéutica con valerato de betametasona al o,1\%. Al terminar el tratamiento se observó disminución de la induración y a los nueve meses la lesión se había resuelto por completo, desapareciendo la induración, la atrofia y las telangiectasias, y sin presentarse ningún efecto adverso. Dos años después de haber finalizado el tratamiento no había presentado recurrencias. Este agente puede ser una alternativa eficaz a los esteroides tópicos ${ }^{43}$.

Calcipotriol en combinación con betametasona: el mecanismo de eficacia de la combinación de calcipotriol y dipropionato de betametasona es doble, ya que ambos componentes inhiben la proliferación de fibroblastos y actúan como inmunomoduladores de citocinas, disminuyendo la fibrosis y la inflamación. En el 2007 se reportó la primera serie de casos que mostró mejoría en cinco de seis pacientes con morfea, después de la aplicación de esta combinación una o dos veces al día. Los autores recomiendan este medicamento como una opción efectiva en el tratamiento de esta entidad y proponen realizar estudios controlados para comparar este agente con otras opciones tópicas ${ }^{44}$.

Imiquimod: se ha visto que este medicamento es inductor del interferón gamma, un inhibidor del factor de crecimiento transformante beta, molécula implicada en la fibrosis de esta entidad. En un reporte de doce casos tratados diariamente con imiquimod en crema al $5 \%$, se demostró disminución de la induración, tanto clínica como histológica, del eritema y de la hipopigmentación, en todos los pacientes con morfea después de seis meses de haber iniciado el tratamiento. Solo se presentó una leve irritación en el sitio de la aplicación, sin necesidad de descontinuar el tratamiento. Es la primera serie de casos en que se describe el uso exitoso del imiquimod en el tratamiento de la morfea ${ }^{45}$.

En dos reportes de casos publicados en el 2009, se demostró la efectividad de imiquimod en crema al 5 $\%$ durante cinco días consecutivos a la semana por un periodo de 16 semanas en pacientes con morfea, sin observarse efectos secundarios locales o sistémicos significativos, excepto por ligero prurito y eritema local. Los pacientes fueron seguidos por 18 meses y, hasta ese momento, no habian presentado recidivas ${ }^{46}$.

En el 2011 se publicó un estudio prospectivo sobre la eficacia y la seguridad del imiquimod en crema al $5 \%$ en nueve niños con morfea en placa; se observó una disminución significativa de la hipopigmentación, la induración, el eritema y las telangiectasias, lo que sugiere que es un tratamiento efectivo, seguro y bien tolerado en la edad pediátrica, sin embargo, se justifican más estudios prospectivos $^{47}$.

\section{Sistémico}

Esteroides sistémicos: se han utilizado en esta entidad por sus efectos inmunomoduladores y antiinflamatorios. El uso de los esteroides orales como monoterapia en esta entidad, se evaluó en un estudio publicado en 1994, en el cual se reportó mejoría de los diecisiete pacientes que incluyó el estudio; sin embargo, su limitación fue no haber evaluado las recaídas después de suspender el tratamiento ${ }^{48}$.

En otro estudio, retrospectivo, en que se evaluaron 28 adultos con formas graves de morfea tratados con esteroides orales (prednisona entre 0,3 y $1 \mathrm{mg} / \mathrm{kg}$ al día), se demostró que la administración de este medicamento puede ser eficaz en el tratamiento de formas serias de morfea en adultos. Sin embargo, la mitad de los pacientes recayó después de suspender el tratamiento; esto indica que se requiere una alternativa terapéutica como el metotrexato al finalizar la administración de esteroides o usar estos dos agentes en combinación desde el principio, para evitar recurrencias ${ }^{48}$.

Metotrexato: el mecanismo de acción a dosis bajas de este medicamento en la esclerodermia localizada, no ha sido completamente entendido. Este agente tiene 
efectos cruciales en la cascada de eventos iniciados por citocinas como la IL-1, la IL-6 y el factor de necrosis tumoral. En la morfea y la esclerosis sistémica, se ha visto un aumento en los niveles séricos de IL-2, IL-4 e IL-6, y la disminución de estos niveles producida por el metotrexato, es paralela a la mejoría clínica de la esclerosis cutánea. Teniendo en cuenta todos estos efectos, se ha visto que este medicamento puede ser una alternativa terapéutica efectiva en estos pacientes ${ }^{49}$.

En un estudio publicado en 1998, se evaluó el efecto de dosis bajas de metotrexato durante 24 semanas, en la morfea generalizada. Se incluyeron nueve pacientes y se les administró metotrexato a dosis de $15 \mathrm{mg}$ por semana. Al finalizar el estudio, se observó una mejoría clínica significativa, sin reportarse efectos adversos graves. Estos resultados sugieren un efecto benéfico del metotrexato en la morfea generalizada ${ }^{50}$.

Se evaluó la eficacia a largo plazo (media de 6,6 años) del tratamiento con metotrexato en 17 pacientes con morfea lineal, en un estudio retrospectivo publicado en el 2013. Todos los pacientes mejoraron, y se concluyó que este agente es eficaz para lograr la inactividad de la enfermedad en niños y adultos jóvenes con morfea lineal; sin embargo, muchos pueden necesitar más de un ciclo de tratamiento ${ }^{51}$.

Combinación de esteroides sistémicos y metotrexato: en un estudio del 2000 se determinaron la efectividad y la tolerabilidad del metotrexato $(0,3$ a $0,6 \mathrm{mg} /$ $\mathrm{kg}$ por semana) y de los esteroides sistémicos (pulsos intravenosos de metilprednisolona de $30 \mathrm{mg} / \mathrm{kg}$ por tres días al mes durante tres meses), en diez pacientes con esclerodermia localizada activa. Uno suspendió el tratamiento con metotrexato y los nueve restantes presentaron una buena reacción clínica ${ }^{52}$.

En el 2005 se publicó un estudio piloto prospectivo abierto, sin asignación aleatoria, en que se evaluó la eficacia de los esteroides en pulsos (metilprednisolona venosa, $1 \mathrm{~g}$ durante tres días cada mes, durante mínimo seis meses) combinados con metotrexato oral ( $15 \mathrm{mg}$ por semana), en 15 pacientes con esclerodermia localizada grave. Uno descontinuó el tratamiento y los 14 restantes presentaron una mejoría significativa de los signos de actividad clínica, sin presentar efectos adversos. Este esquema de tratamiento es seguro y efectivo, y debe considerarse en casos graves de morfea cuando los tratamientos convencionales han fallado ${ }^{53}$.

En un estudio retrospectivo publicado en el 2006, se evaluaron la eficacia y la tolerabilidad del tratamiento combinado de esteroides sistémicos con metotrexato, en 34 niños con morfea. Todos presentaron una mejoría clínica significativa. En 16 se suspendió el tratamiento cuando la enfermedad se consideró clínicamente in- activa, pero 7 (44\%) de estos pacientes presentaron recaída, necesitando repetir el tratamiento. Este tratamiento combinado se considera seguro y benéfico en este grupo etario; sin embargo, por el riesgo de recaída después de suspenderlo, los autores recomiendan un seguimiento clínico estricto ${ }^{54}$.

En el 2008 se publicó otro estudio retrospectivo en el cual se observó la evolución de diez niños mayores de 14 años con morfea, que recibieron tratamiento sistémico con esteroides sistémicos, seguido de un tratamiento de mantenimiento con metotrexato. Ocho pacientes presentaron mejoría, pero, de estos, tres recayeron luego de suspender el tratamiento, requiriendo un segundo ciclo al cual reaccionaron muy bien. El tratamiento fue bien tolerado y se acompañó de pocos efectos secundarios 55 .

En el 2012, se publicó un estudio, en el que se hizo seguimiento por cuatro años a 58 pacientes de una cohorte de 65 pacientes con morfea juvenil, y se observó que el tratamiento a largo plazo con metotrexato, era benéfico y bien tolerado ${ }^{56}$.

En este mismo año se publicó un estudio prospectivo en el cual se observó la reacción al tratamiento combinado de esteroides orales y metotrexato, en 36 niños con esclerodermia de moderada a grave. Treinta y dos completaron el estudio y todos presentaron mejoría clínica significativa, sin presentar recurrencia al disminuir la dosis o cambiar la vía de administración de los medicamentos ${ }^{57}$.

Micofenolato mofetil: es un inhibidor selectivo de la síntesis de novo de las purinas, al producir una inhibición específica sobre la proliferación de los linfocitos $\mathrm{T}$ y B. Cada vez más se emplea en enfermedades de carácter autoinmunitario, como la nefritis lúpica, la psoriasis, el liquen plano, el pénfigo y el pioderma gangrenoso. Recientemente, en estudios in vitro se ha demostrado que este medicamento también inhibe las células del músculo liso, los fibroblastos, células cuya actividad está aumentada en enfermedades como la esclerodermia, la enfermedad crónica de injerto contra huésped y la nefropatía crónica. Además, se ha demostrado que este agente inhibe la expresión de colágeno de tipo I, potenciando así la expresión de MMP-1, molécula que está disminuida en los procesos de fibrosis, alterando las funciones migratorias de los fibroblastos. Por lo anterior, se ha planteado la hipótesis de una acción antifibrótica directa de este medicamento, además de las propiedades inmunosupresoras que se le conocen ${ }^{58}$.

En un estudio retrospectivo, publicado en el 2009, se evaluó la eficacia de este tratamiento en 10 pacientes con morfea juvenil grave resistente al tratamiento combinado (esteroides sistémicos y metotrexato oral) o casos de morfea con manifestaciones extracutáneas se- 
rias. Se demostró mejoría clínica en todos y solo un paciente presentó efectos gastrointestinales, sin requerir la suspensión del fármaco. Concluyeron que este agente parece ser eficaz en detener la progresión de la enfermedad grave o resistente a otros medicamentos y es bien tolerado ${ }^{58}$.

Calcitriol: se ha visto que el calcitriol inhibe indirectamente la secreción de interleucina 2 al inhibir las células T activadas ${ }^{42}$. Además, se ha observado que puede inhibir el crecimiento de los fibroblastos dérmicos humanos y regula su diferenciación. Por lo tanto, la efectividad terapéutica de este medicamento puede estar mediada por el efecto directo que ejerce sobre los fibroblastos y la síntesis de colágeno o al influir en la respuesta inmunitaria ${ }^{42,59,60}$.

En 1999 se publicó un estudio prospectivo abierto, en que se evaluó la efectividad del calcitriol oral, en tres pacientes con morfea generalizada. Después del tratamiento, se observó una remisión clínicamente significativa, incluyendo mejoría en los arcos de movimiento de las articulaciones. Este efecto permaneció durante un año después de haber suspendido el tratamiento. En este estudio se sugiere que este agente se puede utilizar en el tratamiento de la morfea generalizada ${ }^{59}$.

En un estudio de asignación aleatoria, controlado con placebo, publicado en el 2000, se evaluó la efectividad del calcitriol oral durante nueve meses, en 27 pacientes, 20 con esclerodermia localizada y 7 con esclerosis sistémica. Al finalizar el estudio, la puntuación de la piel en los pacientes con morfea no mostró diferencias significativas entre los dos grupos, y en el grupo de los pacientes con esclerosis sistémica, por tratarse de una muestra pequeña y poco representativa, los autores no lograron sacar conclusiones. Finalmente, concluyeron que el calcitriol no fue más eficaz que el placebo en la esclerodermia localizada ${ }^{60}$.

\section{Físicas}

Fototerapia: la introducción de la fotoquimioterapia (PUVA) en 1994 y de la fototerapia UVA 1 (340 a $400 \mathrm{~nm}$ ) en 1995 para la esclerodermia localizada, ha mejorado considerablemente las opciones terapéuticas en esta entidad. La meta principal con la fototerapia es eliminar las lesiones inflamatorias y lograr un máximo reblandecimiento de las lesiones, para evitar posibles complicaciones futuras ${ }^{61,62}$.

En un artículo de revisión publicado en el 2005, se recolectaron los datos sobre la eficacia de diferentes protocolos de tratamiento en esta entidad, como UVA1 (340-400 nm), UVA de banda ancha a dosis bajas (10-20 $\mathrm{J} / \mathrm{cm}^{2}$ ), PUVA baños, PUVA crema y PUVA oral.
Los autores concluyen que tanto la UVA1 y como la fotoquimioterapia (PUVA) se pueden utilizar en esta enfermedad, hasta que se demuestre una superioridad significativa de alguna de ellas sobre la otra. En cuanto a las tasas de recurrencia, los autores refieren que las nuevas lesiones son comunes, pudiéndose necesitar nuevos ciclos de fototerapia ${ }^{61}$.

En un estudio publicado en el 2006, se evaluó la eficacia de dosis bajas de UVA1 (340-400 nm), dosis media de UVA 1 y fototerapia UVB de banda estrecha, en el tratamiento de la morfea. Se incluyeron 74 pacientes, los cuales fueron distribuidos al azar en tres grupos. El grupo de la UVB de banda estrecha mostró una disminución estadísticamente significativa en el engrosamiento de la piel. El grupo de dosis media de UVA1 tuvo una disminución estadísticamente significativa en el espesor de la lesión por ecografía. Y cuando se compararon los grupos de tratamiento, el grupo de dosis media de UVA1 tenía una mejoría estadísticamente significativa en comparación con la UVB de banda estrecha, pero no cuando se comparaba con la dosis baja de UVA1. Tanto la UVA 1 como la UVB de banda estrecha mostraron una mejoría equivalente, concluyéndose que la UVB de banda estrecha podría ser una opción de tratamiento seguro, eficaz y de fácil acceso en esta entidad ${ }^{62}$.

En otro estudio publicado en el 2010, se demostraron buenos resultados del tratamiento a corto y a largo plazo con UVA1, en 30 pacientes con morfea ${ }^{63}$.

En cuanto a la fotoquimioterapia, en un estudio publicado en el 2008 se evaluaron 13 pacientes con morfea que se trataron con PUVA (11 con psolareno oral y 2 con psolareno tópico). Al finalizar el tratamiento, 11 de ellos presentaban una mejoría clínica importante ${ }^{64}$.

En el 2013 se publicó un estudio retrospectivo sobre la efectividad de PUVA en baño (tres sesiones a la semana en una solución de $0,2 \mathrm{mg}$ por litro de 8-metoxipsoraleno, seguido de irradiación con UVA), en 28 pacientes con esclerodermia localizada. Once pacientes (39\%) mostraron remisión completa, 14 (50 \%) presentaron ablandamiento y regresión parcial de las lesiones, y 3 $(10,7 \%)$ no presentaron ninguna mejoría. Los autores concluyen que, en su experiencia, la fotoquimioterapia en baños es eficaz y bien tolerada, pudiéndose considerar como uno de los modalidades terapéuticas de primera línea ${ }^{65}$.

En conclusión, la UVA1, la UVA banda ancha, la PUVA y la UVB de banda estrecha, han demostrado eficacia en el tratamiento de la morfea. Sin embargo, la UVA1 a altas dosis es probablemente la opción más eficaz y con menos efectos adversos en comparación con la fotoquimioterapia, pero, por la poca disponibilidad de esta longitud de onda en el medio médico, se recomienda llevar a cabo más estudios con UVA de banda ancha y con UVB 
de banda estrecha, para así confirmar su eficacia en esta entidad y evitar los efectos adversos que se pueden presentar con la PUVA ${ }^{34}$.

\section{Nuevas alternativas terapéuticas}

Productos biológicos: se reportó un caso exitoso de uso de infliximab en una mujer con morfea generalizada recalcitrante, con resultados excelentes después de cuatro meses de tratamiento. Sin embargo, se requieren estudios controlados para confirmar estos datos ${ }^{66}$.

En el 2011 se publicó un reporte de caso sobre el uso exitoso del imatinib (200 $\mathrm{mg}$ al día por seis meses) en un hombre con morfea generalizada, la cual comprometía la movilidad de sus articulaciones y había sido resistente a múltiples tratamientos sistémicos. Sin embargo, seis meses después de la suspensión del tratamiento reaparecieron las lesiones inflamatorias. Los autores sugieren más estudios para comprobar su eficacia, la dosis y la duración del tratamiento ${ }^{67}$.

El uso de abatacept, un inhibidor de la activación de las células T, también se ha descrito en la literatura científica como un tratamiento promisorio para la morfea profunda generalizada, así como lo demuestran dos reportes de caso publicados en el $2011^{68}$.

A pesar de los datos anteriores, en la literatura médica hay dos reportes de caso de esclerodermia localizada como efecto adverso del tratamiento con adalimumab: uno en una mujer de 17 años que estaba recibiendo este medicamento para el tratamiento de la enfermedad de Crohn, y otro, en un hombre de 37 años de edad con espondilitis anquilosante ${ }^{69,70}$.

Rellenos con ácido hialurónico: su uso ha sido efectivo, especialmente en casos de morfea lineal, como lo demuestran dos reportes de caso, uno publicado en el 2011 donde se utilizó en un hombre de 20 años con morfea en golpe de sable que comprometía la hemicara izquierda, y el reporte más reciente, publicado en el 2013, en el que se usó en una mujer caucásica de 44 años de edad que también tenía una morfea en golpe de sable $^{71,72}$.

\section{Esquemas de tratamiento que recomienda}

\section{la literatura}

Morfea en placas limitada: estos pacientes tienen bajo riesgo de deformidad facial, de alteración en extremidades y de contracturas, por lo que idealmente deben ser tratados con agentes tópicos. Se debe iniciar con tacrolimus tópico, dos veces al día. Si después de ocho semanas no se observa mejoría, se puede cambiar el tratamiento por cualquiera de las siguientes opciones: fototerapia limitada a la lesión (UVA1, PUVA en baños, UVB de banda estrecha), calcipotrieno ocluido dos veces al día, calcipotrieno más dipropionato betametasona, una o dos veces al día, o imiquimod tópico, tres veces por semana ${ }^{34,38}$.

Morfea lineal: los pacientes con morfea lineal de la cabeza, cuello o extremidades tienen un alto riesgo de deformidad facial, contracturas de las extremidades y diferencia en su longitud; por lo tanto, deben recibir tratamiento sistémico. Idealmente, se debe iniciar con la combinación de metotrexato y esteroides sistémicos. Si el paciente no presenta mejoría después de dos a tres meses, el tratamiento de elección es la fototerapia según la disponibilidad, ya sea UVA1 o UVA, PUVA o UVB de banda estrecha. Si la fototerapia no está disponible o hay contraindicaciones para administrarla, se recomienda el micofenolato de mofetilo sobre el cual se tiene poca evidencia, aunque no menor que sobre otros inmunosupresores sistémicos, como la ciclosporina, la ciclofosfamida, el imatinib, la d-penicilamina, los inhibidores del factor de necrosis tumoral alfa o la fotoféresis extracorpórea, con un perfil de seguridad más alto. El micofenolato de mofetilo es la opción terapéutica de elección cuando ha fracasado el metotrexato en combinación con los esteroides sistémicos ${ }^{34,38}$.

Morfea generalizada: en este tipo de morfea, el tratamiento depende de la presentación clínica. Cuando no hay compromiso de las articulaciones, la fototerapia (UVA1 o UVA, PUVA, UVB de banda estrecha) es la primera opción terapéutica, ya que tiene un perfil de seguridad mayor en comparación con la combinación de metotrexato y esteroides sistémicos. Sin embargo, si el paciente no ha mejorado después de dos a tres meses, se puede pensar en la opción de metotrexato más esteroides sistémicos; $\mathrm{y}$, si después de un período de dos a tres meses no se ve mejoría, se recomienda iniciar el micofenolato de mofetilo. Si se presenta un paciente con morfea generalizada profunda o que comprometa las articulaciones, se debe tratar como la morfea lineal ${ }^{34,38}$.

\section{Conclusiones}

La morfea, o esclerodermia localizada, es una entidad poco común y con múltiples puntos de controversia, como lo ha sido la clasificación, las medidas que evalúan la respuesta terapéutica y el protocolo de tratamiento; sin embargo, cada vez está más clara su patogénesis, lo cual ha logrado tener un impacto en los nuevos tratamientos para los casos más serios y recalcitrantes. Es importante que estos pacientes, aparte de la evalua- 
ción dermatológica, sean manejados por un grupo multidisciplinario, que incluya especialistas en ortopedia, fisiatría y fisioterapia, para evitar secuelas y disminuir la morbilidad que conlleva este tipo de lesiones.

\section{Referencias}

1. Laborde HA, Young P. Historia de la esclerosis sistémica. Gac Méd Méx. 2012;148:201-8.

2. Fett N, Werth VP. Update on morphea: Part I. Epidemiology, clinical presentation, and pathogenesis. J Am Acad Dermatol. 2011;64:217-28.

3. Takehara K, Sato S. Localized scleroderma is an autoimmune disorder. Rheumatology. 2005;44:274-9.

4. Bielsa I. Update on the classification and treatment of localized scleroderma. Actas Dermosifiliogr. 2013;104:654-66.

5. Peterson LS, Nelson AM, Su WP, Mason T, O’Fallon WM, Gabriel SE. The epidemiology of morphea (localized scleroderma) in Olmsted County, 1960-1993. J Rheumatol. 1997;24:73-80.

6. Christen-Zaech S, Hakim MD, Afsar FS, Paller AS. Pediatric morphea (localized scleroderma): Review of 136 patients. J Am Acad Dermatol. 2008;59:385-96.

7. Kreuter A. Localized scleroderma. Dermatol Ther. 2012;25:135-47.

8. Leitenberger JJ, Cayce RL, Haley RW, Adams-Huet B, Bergstresser PR, Jacobe HT. Distinct autoimmune syndromes in morphea: A review of 245 adult and pediatric cases. Arch Dermatol. 2009;145:545-50.

9. Canady J, Karrer S, Fleck M, Bosserhoff AK. Fibrosing connective tissue disorders of the skin: Molecular similarities and distinctions. J Dermatol Sci. 2013;70:151-8.

10. Valanciene G, Jasaitiene D, Valiukeviciene S. Pathogenesis and treatment modalities of localized scleroderma. Medicina (Kaunas). 2010;46:649-56.

11. Yaqub A, Chung L, Rieger KE, Fiorentino DF. Localized cutaneous fibrosing disorders. Rheum Dis Clin North Am. 2013;39:347-64.

12. Kurzinski K, Torok KS. Cytokine profiles in localized scleroderma and relationship to clinical features. Cytokine. 2011;55:157-64.

13. Zulian F, Athreya BH, Laxer R, Nelson AM, Feitosa de Oliveira SK, Punaro MG, Cuttica R, et al. Juvenile localized scleroderma: Clinical and epidemiological features in 750 children. An international study. Rheumatology (Oxford). 2006;45:614-20.

14. Bleasel NR, Stapleton KM, Commens C. Radiation-induced localized scleroderma in breast cancer patients. Australas J Dermatol.1999;40:99-102.

15. Herrmann T, Gunther C, Csere P. Localized morphea -a rare but significant secondary complication following breast cancer radiotherapy. Case report and review of the literature on radiation reaction among patients with scleroderma/morphea. Strahlenther Onkol. 2009;185:603-7.

16. Peroni A, Zini A, Braga V, Colato C, Adami S, Girolomoni G. Druginduced morphea: Report of a case induced by balicatib and review of the literature. J Am Acad Dermatol. 2008;59:125-9.

17. Eisendle K, Grabner T, Zelger B. Morphoea: A manifestation of infection with Borrelia species? Br J Dermatol. 2007;157:1189-98.

18. Peterson LS, Nelson AM, Su WP. Classification of morphea (localized scleroderma). Mayo Clin Proc. 1995;70:1068-76.

19. Laxer RM, Zulian F.Localized scleroderma. Curr Opin Rheumatol. 2006;18:606-13.
20. Kreuter A, Krieg T, Worm M, Wenzel J, Gambichler T, Kuhn A, et al. AWMF Guideline No. 013/066. Diagnosis and therapy of circumscribed scleroderma. J Dtsch Dermatol Ges. 2009;6:S1-14.

21. Weibel L, Harper JI. Linear morphoea follows Blaschko's lines. Br J Dermatol. 2008;159:175-81.

22. Duymaz A, Karabekmez FE, Keskin M, Tosun Z. Parry-Romberg syndrome: Facial atrophy and its relationship with other regions of the body. Ann Plast Surg. 2009;63:457-61.

23. Tollefson MM, Witman PM.En coup de sabre morphea and ParryRomberg syndrome: A retrospective review of 54 patients. J Am Acad Dermatol. 2007;56:257-63.

24. Parodi PC, Riberti C, Draganic Stinco D, Patrone P, Stinco G. Squamous cell carcinoma arising in a patient with long-standing pansclerotic morphea. Br J Dermatol. 2001;144:417-9.

25. Wollina U, Buslau M, Weyers W. Squamous cell carcinoma in pansclerotic morphea of childhood. Pediatr Dermatol. 2002;19:151-4.

26. Wollina U, Buslau M, Heinig B, Petrov I, Unger E, Kyriopoulou E, et al. Disabling pansclerotic morphea of childhood poses a high risk of chronic ulceration of the skin and squamous cell carcinoma. Int J Low Extrem Wounds. 2007;6:291-8.

27. Blaszczyk M, Królicki L, Krasu M, Glinska O, Jablonska S. Progressive facial hemiatrophy: Central nervous system involvement and relationship with scleroderma en coup de sabre. J Rheumatol. 2003;30:1997-2004.

28. Zannin ME, Martini G, Athreya BH, Russo R, Higgins G, Vittadello $\mathrm{F}$, et al. Ocular involvement in children with localized scleroderma: A multi-centre study. Br J Ophthalmol. 2007;91:1311-4.

29. 29. Torres JE, Sánchez JL. Histopathological differentiation between localized and systemic scleroderma. Am J Dermatopathol. 1998;20:242-5.

30. Walters R, Pulitzer M, Kamino H. Elastic fiber pattern in scleroderma/ morphea. J Cutan Pathol. 2009;36:952-7.

31. Vierra E, Cunningham BB. Morphea and localized scleroderma in children. Semin Cutan Med Surg. 1999;18:210-25.

32. Yimane K, Ihn H, Kubo M, Asano Y, Yazawa N, Tamaki K. AntiU3 snRNP antibodies in localized scleroderma. Ann Rheum Dis. 2001;60:1157-8.

33. Sommer A, Gambichler T, Bacharach-Buhles M, von Rothenburg T, Altmeyer P, Kreuter A. Clinical and serological characteristics of progressive facial hemiatrophy: A case series of 12 patients. J Am Acad Dermatol. 2006;54:227-33.

34. Fett N, Werth VP. Update on morphea: Part II. Outcome measures and treatment. J Am Acad Dermatol. 2011;64:231-42.

35. Arkachaisri T, Vilaiyuk S, Torok KS, Medsger TA Jr. Development and initial validation of the localized scleroderma skin damage index and physician global assessment of disease damage: A proof-of-concept study. Rheumatology. 2010;49:373-81.

36. Weibel L, Howell KJ, Visentin MT, Rudiger A, Denton CP, Zulian F, et al • Laser Doppler flowmetry for assessing localized scleroderma in children. Arthritis Rheum. 2007;56:3489-95.

37. Zulian F, Meneghesso D, Grisan E, Vittadello F, Belloni Fortina A, Pigozzi B, et al. A new computerized method for the assessment of skin lesions in localized scleroderma. Rheumatology. 2007;46:856-60.

38. Fett NM. Morphea: Evidence-based recommendations for treatment. Indian J Dermatol Venereol Leprol. 2012;78:135-41.

39. Mancuso G, Berdondini RM. Localized scleroderma: Response to occlusive treatment with tacrolimus ointment. $\mathrm{Br}$ J Dermatol. 2005;152:180-2. 
40. Stefanaki C, Stefanaki K, Kontochristopoulos G, Antoniou C, Stratigos A, Nicolaidou E, et al. Topical tacrolimus $0.1 \%$ ointment in the treatment of localized scleroderma. An open label clinical and histological study. J Dermatol. 2008;35:712-8.

41. Kroft EB, Groeneveld TJ, Seyger MM, de Jong EM. Efficacy of topical tacrolimus $0.1 \%$ in active plaque morphea randomized, double-blind, emollient-controlled pilot study. Am J Clin Dermatol. 2009;10:181-7.

42. Cunningham BB, Landells ID, Langman C, Sailer DE, Paller AS.Topical calcipotriene for morphea/linear scleroderma. J Am Acad Dermatol. 1998;39:211-5.

43. Tay YK. Topical calcipotriol ointment in the treatment of morphea. J Dermatolog Treat. 2003;14:219-21.

44. Dytoc MT, Kossintseva I, Ting PT. First case series on the use of calcipotriol- betamethasone dipropionate for morphoea. Br J Dermatol. 2007;157:615-8.

45. Dytoc M, Ting PT, Man J,Sawyer D, Fiorillo L. First case series on the use of imiquimod for morphoea. Br J Dermatol. 2005;153:815-20.

46. Campione E, Paternó EJ,Diluvio L, Orlandi A, Bianchi L, Chimenti S. Localized morphea treated with imiquimod $5 \%$ and dermoscopic assessment of effectiveness. J Dermatolog Treat. 2009;20:10-3.

47. Pope E, Doria AS, Theriault M, Mohanta A, Laxer RM. Topical imiquimod $5 \%$ cream for pediatric plaque morphea: A prospective, multiple-baseline, open-label pilot study. Dermatology. 2011;223:363-9.

48. Amy de la Bretéque M, Rybojad M, Cordoliani F, Petit A, Juillard C, Flageul B, et al. Relapse of severe forms of adult morphea after oral corticosteroid treatment. J Eur Acad Dermatol Venereol. 2013;27:1190-1.

49. Zulian F, Martini G, Vallongo C, Vittadello F, Falcini F, Patrizi A, et al. Methotrexate treatment in juvenile localized scleroderma: A randomized, double-blind, placebo-controlled trial. Arthritis Rheum. 2011;63:1998-2006.

50. Seyger MM, van den Hoogen FH, de Boo T, de Jong EM. Low-dose methotrexate in the treatment of widespread morphea. J Am Acad Dermatol. 1998;39:220-5.

51. Koch SB, Cerci FB, Jorizzo JL, Krowchuk D. Linear morphea: A case series with long-term follow-up of young, methotrexatetreated patients. J Dermatolog Treat. 2013;24:435-8.

52. Uziel Y, Feldman BM, Krafchik BR, Yeung RS, Laxer RM.Methotrexate and corticosteroid therapy for pediatric localized scleroderma. J Pediatr. 2000;136:91-5.

53. Kreuter A, Gambichler T, Breuckmann F, Rotterdam S, Freitag M, Stuecker M, et al. Pulsed high-dose corticosteroids combined with low-dose methotrexate in severe localized scleroderma. Arch Dermatol. 2005;141:847-52.

54. Weibel L, Sampaio MC, Visentin MT, Howell Kj, Woo P, Harper JI. Evaluation of methotrexate and corticosteroids for the treatment of localized scleroderma (morphoea) in children. Br J Dermatol. 2006;155:1013-20.

55. Cox D, O’Regan G, Collins S, Byrne A, Irvine A, Watson R. Juvenile localized scleroderma: A retrospective review of response to systemic treatment. Ir J Med Sci. 2008;177:343-6.

56. Zulian F, Vallongo C, Patrizi A, Belloni-Fortina A, Cutrone M, Alessio M, et al. A long-term follow-up study of methotrexate in juvenile localized scleroderma (morphea). J Am Acad Dermatol. 2012;67:1151-6.
57. Torok KS, Arkachaisri T. Methotrexate and corticosteroids in the treatment of localized scleroderma: A standardized prospective longitudinal single center study. J Rheumatol. 2012;39:286-94.

58. Martini G, Ramanan AV, Falcini F, Girschick H, Goldsmith DP, Zulian F.Successful treatment of severe or methotrexate-resistant juvenile localized scleroderma with mycophenolate mofetil. Rheumatology. 2009;48:1410-3.

59. Caca-Biljanovska NG, VIckova-Laskoska MT, Dervendi DV,Pesic NP, Laskoski DS. Treatment of generalized morphea with oral 1,25-dihydroxyvitamin D3. Adv Exp Med Biol. 1999;455:299-304.

6o. Hulshof MM, Bouwes JN, Bergman W, Masclee AA, Heickendorff L, Breedveld FC, et al. Double-blind, placebo-controlled study of oral calcitriol for the treatment of localized and systemic scleroderma. J Am Acad Dermatol. 2000;43:1017-23.

61. Brenner M, Herzinger T, Berking C, Plewig G, Degitz K. Phototherapy and photochemotherapy of sclerosing skin diseases. Photodermatol Photoimmunol Photomed. 2005;21:157-65.

62. Kreuter A, Hyun J, Stucker M, Sommer A, Altmeyer P, Gambichler T. A randomized controlled study of low-dose UVA1, mediumdose UVA1, and narrowband UVB phototherapy in the treatment of localized scleroderma. J Am Acad Dermatol. 2006;54:440-7.

63. Andres C, Kollmar A, Mempel M, Hein R, Ring J, Eberlein B. Successful ultraviolet A1 phototherapy in the treatment of localized scleroderma: A retrospective and prospective study. Br J Dermatol. 2010;162:445-7.

64. Usmani N, Murphy A, Veale D, Goulden V, Goodfield M. Photochemotherapy for localized morphoea: Effect on clinical and molecular markers. Clin Exp Dermatol. 2008;33:698-704.

65. Pavlotsky F, Sakka N, Lozinski A, Barzilai A. Bath psoralen-UVA photochemotherapy for localized scleroderma: Experience from a single institute. Photodermatol Photoimmunol Photomed. 2013;29:247-52.

66. Diab M, Coloe JR, Magro C, Bechtel MA. Treatment of recalcitrant generalized morphea with infliximab. Arch Dermatol. 2010;146:601-4.

67. Moinzadeh P, Krieg T, Hunzelmann N. Imatinib treatment of generalized localized scleroderma (morphea). J Am Acad Dermatol. 2010;63:102-4.

68. Stausbol-GronB, Olesen AB, Deleuran B, Deleuran MS. Abatacept is a promising treatment for patients with disseminated morphea profunda: Presentation of two cases. Acta Derm Venereol. 2011;91:686-8.

69. Mattozzi C, Richetta AG, Cantisani C, Giancristoforo S, D’Epiro S, Gonzalez Serva A, et al. Morphea, an unusual side effect of antiTNF-alpha treatment. Eur J Dermatol. 2010;20:400-1.

70. Ramírez J, Hernández MV, Galve J, Cañete JD, Sanmartí R. Morphea associated with the use of adalimumab: A case report and review of the literature. Mod Rheumatol. 2012;22:602-4.

71. Choksi AN, Orringer JS. Linear morphea-induced atrophy treated with hyaluronic acid filler injections. Dermatol Surg. 2011;37:880-3.

72. Thareja SK, Sadhwani D, Alan Fenske N.En coup de sabre morphea treated with hyaluronic acid filler. Report of a case and review of the literature. Int J Dermatol. 2013;29:1-4. 